\title{
Serum vitamin D level - the effect on the clinical course of psoriasis
}

\author{
Beata Bergler-Czop, Ligia Brzezińska-Wcisło
}

Department of Dermatology, School of Medicine, Medical University of Silesia, Katowice, Poland

Adv Dermatol Allergol 2016; XXXIII (6): 445-449

DOI:10.5114/ada.2016.63883

\begin{abstract}
Introduction: Psoriasis is a hyperproliferative disorder of the skin, and vitamin D analogs are widely used in its treatment. It is evident that ultraviolet radiation enables vitamin $\mathrm{D}_{3}$ (cholecalciferol) formation in the epidermis, and this product is further converted into the active metabolites 25 -hydroxycholecalciferol and 1,25-hydroxycholecalciferol, which exert several important effects on the skin. The disruption in proper functioning of the skin which occurs in psoriasis leads to a loss of capacity for cutaneous synthesis of vitamin $D_{3}$. In consequence, it activates a vicious circle that impairs homeostasis of the skin and results in a progressive decrease in the level of vitamin $D$ in the whole human body.

Aim: To estimate the prevalence of vitamin D serum deficiency in patients with psoriasis and analyse the association of vitamin D food intake with clinical features.

Material and methods: Forty adults with psoriasis and 40 healthy subjects (control group) were recruited. Psoriasis plaques were diagnosed and evaluated by the PASI scale. Collected blood samples enabled measurement of serum vitamin $\mathrm{D}$ level by assessment with the immunoenzyme technique.

Results: The analysis with the Mann-Whitney $U$ test revealed a statistically significant difference in 25-hydroxycholecalciferol level between healthy individuals and patients with psoriasis $(p=0.048)$. In both groups (control and psoriatic) the level of 25-hydroxycholecalciferol was seriously deficient $(<50 \mathrm{nmol} / \mathrm{l})$. There was also a negative correlation of 25-hydroxycholecalciferol serum level with both PASI $(r=-0.43)$ and the duration of psoriasis $(r=-0.53)$. Conclusions: It is necessary to bear in mind that not only the ingestion of food rich in vitamin $D$ is necessary, but also the production of vitamin D with sun exposure. The quantity of 25-hydroxycholecalciferol is very important both in the general population and in patients with psoriasis, because these groups have a distinct metabolism.
\end{abstract}

Key words: psoriasis, 25-hydroxycholecalciferol, serum level.

\section{Introduction}

Psoriasis is a hyperproliferative disorder of the skin, and vitamin $D_{3}$ analogs are widely used in its treatment. There are several agents, such as tacalcitol, calcipotriol and the recently approved maxacalcitol, which are administered either as monotherapy or in combination with topical steroids, such as betamethasone dipropionate, in the treatment of psoriasis. These analogs exert prodifferentiating and antiproliferative effects on keratinocytes, and additionally they manifest important anti-inflammatory properties [1].

The active form of vitamin $D_{3}, 1,25$-dihydroxyvitamin $D_{3}$, is well known for its influence on bones and control of calcium and phosphate homeostasis. It is now evident that $1,25(\mathrm{OH})_{2} \mathrm{D}_{3}$ exerts much more effects in vari- ous tissues which express the vitamin D receptor (VDR) or possess certain enzymes - those necessary for generation of $1,25(\mathrm{OH})_{2} \mathrm{D}_{3}$ by activating hydroxylation of vitamin $D_{3}$ metabolites. It is believed that most tissues have the ability to convert vitamin $\mathrm{D}_{3}$ into its active form, $1,25(\mathrm{OH})_{2} \mathrm{D}_{3}$, which in turn binds to the VDR and forms the $1,25(\mathrm{OH})_{2} \mathrm{D}_{3}$ NVDR complex, which subsequently regulates the expression of several genes. Among special properties of $1,25(\mathrm{OH})_{2} \mathrm{D}_{3}$ are both a prodifferentiating and an antiproliferative influence on normal and cancer cells, as well as some immunomodulatory effects. These aspects are very desirable; however, excessive doses of $1,25(\mathrm{OH})_{2} \mathrm{D}_{3}$ are known to result in hypercalcemia. It could potentially limit the use of $1,25(\mathrm{OH})_{2} \mathrm{D}_{3}$ analogs in any therapy, but synthetic analogs are administered topically, which reduces the risk of calcemic side effects

Address for correspondence: Beata Bergler-Czop, 2a Leśna St, 42-624 Ossy, Poland, phone/fax: +48 3228408 77, e-mail: bettina2@tlen.pl Received: 21.07.2015, accepted: 26.10.2015. 
and simultaneously preserves all beneficial effects of $1,25(\mathrm{OH})_{2} \mathrm{D}_{3}[1-4]$.

There has been a lot of speculation in evaluation of the optimal concentration of vitamin D and a threshold below which a vitamin $D$ insufficiency is recognized. The reference concentration for vitamin $D$ (25-hydroxycholecalciferol) is considered to be 30-50 ng/ml (75-125 nmol/l), while a serum level below $20 \mathrm{ng} / \mathrm{ml}$ (or $50 \mathrm{nmol} / \mathrm{l}$ ) indicates vitamin $D$ deficiency. The problem of vitamin D insufficiency seems to be very common in Europe and the USA, and some estimations suggest its prevalence in the general population to be as high as 50\%. Undoubtedly, the low level of vitamin $D$ leads to a pathological condition with muscle weakness, rickets or osteomalacia, which are complications of poor prognosis [5].

The role of vitamin D is considered to vary, and some evidence suggests vitamin D to be a modulatory factor of the activity in dendritic cells and keratinocytes, or the proliferation in T-cells. All metabolites of vitamin $D$ act via the VDR, which is expressed in many tissues, including the skin. It is evident that ultraviolet radiation enables vitamin $D_{3}$ (cholecalciferol) formation in the epidermis, and this product is further converted to the active metabolites 25-hydroxycholecalciferol and 1,25-hydroxycholecalciferol, which have an influence on cutaneous functions. One of these metabolites, 25-hydroxycholecalciferol, manifests double activity in keratinocytes, by inhibition of their proliferation and enhancement of the maturity. Taking such influence on keratinocytes into consideration, a deficiency in 25-hydroxycholecalciferol could be a risk factor for the development of psoriasis with possible involvement of disturbances in the cellular immune system ( $T$ lymphocytes), as well as in angiogenesis [1, 5, 6]. It is believed that psoriasis could activate a vicious circle with severe impairment of cutaneous functions and, secondarily, a progressive decrease in the level of vitamin $D_{3}$ derivatives in the whole human body, including the skin.

Table 1. Reference intervals for $25(\mathrm{OH})$-vitamin $\mathrm{D}_{3}(\mathrm{ng} / \mathrm{ml})$, males and females

\begin{tabular}{lccc}
\hline Age & $\boldsymbol{N}$ & $\mathbf{2 . 5 \%}$ & $\mathbf{9 7 . 5 \%}$ \\
\hline 0 to $<3$ months & 131 & 5 & 42 \\
\hline 3 to < 6 months & 135 & 9 & 60 \\
\hline 6 months to < 1 year & 147 & 18 & 58 \\
\hline 1 to < 3 years & 394 & 15 & 54 \\
\hline 3 to < 10 years & 619 & 14 & 46 \\
\hline 10 to < 13 years & 286 & 11 & 50 \\
\hline 13 to < 15 years & 275 & 10 & 44 \\
\hline 15 to < 18 years & 390 & 8 & 45 \\
\hline$<18$ years & 421 & 8 & 56 \\
\hline
\end{tabular}

Conversion factor: $1 \mathrm{ng} / \mathrm{ml}=2.5 \mathrm{nmol} / \mathrm{l}, 1 \mathrm{nmol} / \mathrm{l}=0.4 \mathrm{ng} / \mathrm{ml}$.

\section{Aim}

The aim of this study was to estimate the prevalence of vitamin D serum deficiency in patients with psoriasis and perform further analysis of the association of vitamin D food intake with clinical features.

\section{Material and methods}

Forty adults with psoriasis and 40 subjects (healthy control group) were recruited. All patients with psoriasis and all control group subjects agreed to participate in the study. Informed consent was obtained from all subjects. For publication of patient photos, consent to publish was obtained. All the participants were asked to complete the specific questionnaire. The obtained data included age, gender, duration of psoriasis, concomitant diseases and medications. In the group of patients psoriasis was moderate to severe. Psoriasis plaques were diagnosed and evaluated by a specialized medical team with assessment of the Psoriasis Area and Severity Index (PASI). All patients were seen by a dermatologist, who collected data considering the demographic, health status and any other relevant details. Cases were age- and gendermatched for comparison. Collected blood samples enabled measurement of the serum level of a vitamin $D_{3}$ derivative (25-hydroxycholecalciferol) using an immunoenzyme assessment technique $(25(\mathrm{OH})$-Vitamin D Xpress ELISA kit Immundiagnostik AG, Germany). Sensitivity of the method is $6.2 \mathrm{nmol} / \mathrm{l}$, in-series error $6 \%$, inter-series error $7.4 \%$.

All measurements were conducted during winter months to avoid the influence of sun exposure on synthesis of vitamin $D_{3}$ and its derivative 25 -hydroxycholecalciferol (Table 1).

The results were referred to normal serum ranges of 25-hydroxycholecalciferol according to the American Society for Bone and Mineral Research 2011: deficiency (seriously deficient): < $50 \mathrm{nmol} / \mathrm{l}$; insufficiency (deficient): 50-74 nmol/l; sufficiency (adequately supplied): $>75 \mathrm{nmol} / \mathrm{l}$.

\section{Statistical analysis}

Initially, all results obtained from patients and healthy controls were compared using the Shapiro-Wilk test. Subsequently, the Mann-Whitney $U$ test was used to compare the quantitative variables. Correlations were estimated by nonparametric Spearman's rank test. In this study, a $p$-value of $<0.05$ was considered to be statistically significant.

\section{Results}

Results was presented in Tables 2-4.

The analysis with the Mann-Whitney $U$ test revealed a statistically significant difference in 25-hydroxychole- 
Table 2. Serum level of 25-hydroxycholecalciferol, PASI, age and duration of psoriasis in patient group and control group

\begin{tabular}{llccccc}
\hline Parameter & $\boldsymbol{N}$ & Mean & Median & Min. & Max. & SD \\
\hline Study group & 40 & 32.25 & 29.75 & 23.59 & 48.12 & 6.79 \\
\hline PASI & 40 & 10.36 & 11.15 & 1.20 & 28.00 & 6.26 \\
\hline Age & 40 & 41.55 & 41.50 & 18.00 & 76.00 & 14.42 \\
\hline Duration of psoriasis & 40 & 12.89 & 11.50 & 0.50 & 40.00 & 9.01 \\
\hline D_control & 40 & 56.13 & 55.34 & 49.65 & 86.57 & 6.82 \\
\hline
\end{tabular}

Table 3. 25-hydroxycholecalciferol serum level in male group

\begin{tabular}{llccccc}
\hline Group & $\boldsymbol{N}$ & Mean & Median & Min. & Max. & SD \\
\hline Study & 19 & 31.97 & 29.77 & 23.59 & 44.81 & 6.16 \\
\hline D_control & 19 & 57.62 & 56.18 & 50.33 & 86.57 & 8.16 \\
\hline
\end{tabular}

Table 4. 25-hydroxycholecalciferol serum level in female group

\begin{tabular}{lllllll}
\hline Group & $\boldsymbol{N}$ & Mean & Median & Min. & Max. & SD \\
\hline Study & 21 & 32.50 & 28.91 & 24.58 & 48.12 & 7.46 \\
\hline D_control & 21 & 54.77 & 53.55 & 49.65 & 71.23 & 5.17 \\
\hline
\end{tabular}

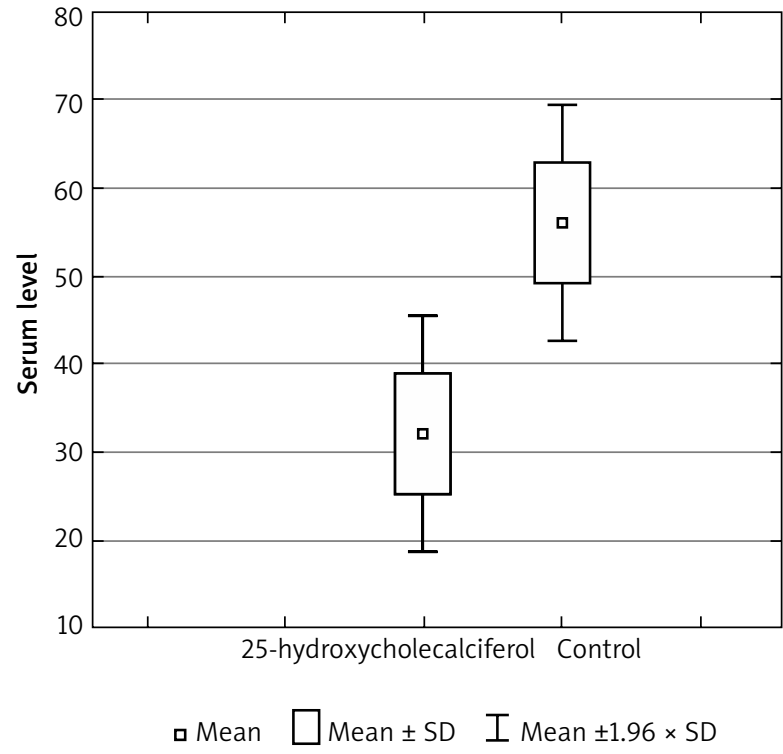

Figure 1. Significant difference between 25-hydroxycholecalciferol serum level in control group and in group of patients with psoriasis $(p=0.048)$

calciferol level between healthy individuals and patients with psoriasis ( $p=0.048$ ) (Figure 1 ). In both groups (control and psoriatic) the level of 25-hydroxycholecalciferol was seriously deficient $(<50 \mathrm{nmol} / \mathrm{l})$. There was also a negative correlation of 25-hydroxycholecalciferol serum level with both PASI $(r=-0.43)$ and the duration of psoriasis $(r=-0.53)$ (Figure 2).

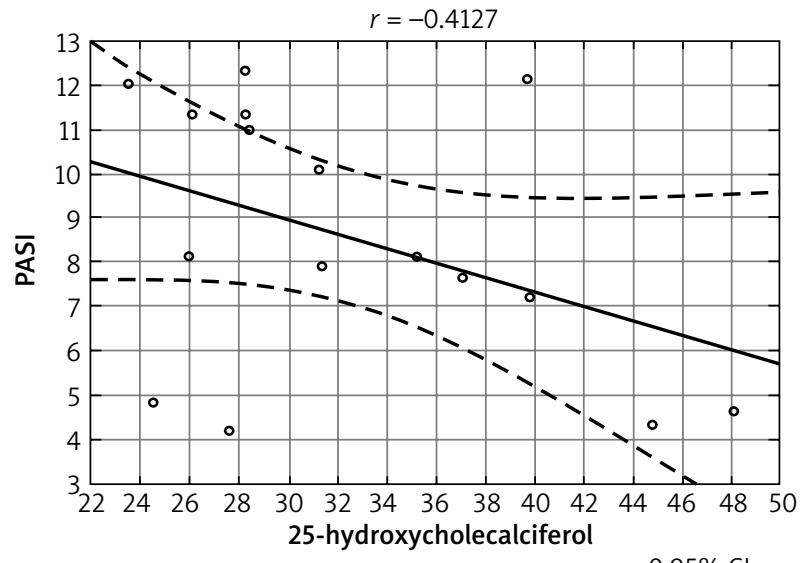

$0.95 \% \mathrm{Cl}$

Figure 2. Negative correlation between 25-hydroxycholecalciferol serum level and PASI $(r=-0.43)$ and psoriasis duration $(r=-0.53)$

\section{Discussion}

Vitamin $D_{3}$ and its analogs exhibit a strong antiproliferative and prodifferentiating influence on both normal and malignant cell types. Several vitamin $D_{3}$ analogs have been approved for the treatment of psoriasis, osteoporosis or secondary hyperparathyroidism, and frequently they comprise the first or second-line treatment option.

The exact mechanism of action of vitamin D analogs still requires further elucidation. Undoubtedly, the reason why specific analogs manifest superagonistic activity in specific tissues remains unknown, although several studies have tried to clarify the mechanisms behind these tissue-specific effects. Moreover, since some cell types 
prefer specific catabolism pathways and enzymes above others, the degradation process may also contribute to the tissue-specific activity of vitamin D analogs. The affinity for the vitamin D binding protein (DBP) may also play a role in the activity of vitamin D analogs, because it regulates the bioavailability of vitamin D for tissues [1, $7,8]$. The relation between 25 -hydroxycholecalciferol and psoriasis has been studied since the 1930s. A chance discovery was reported in 1985 by Morimoto et al., who noticed that the administration of vitamin $D_{3}$ could improve psoriasis in some cases [8]. The new synthetic analogs of vitamin D, such as tacalcitol and calcipotriol, present lower hypercalcemic activity with simultaneously preservation of all biological effects considering the regulation of epidermal cell proliferation and differentiation, the inhibition of angiogenesis and the modulation of cytokine production [9-14].

In our study, the serum level of 25-hydroxycholecalciferol stayed below the normal serum range recommended by the American Society for Bone and Mineral Research, in both groups (controls and patients). The concentration of 25-hydroxycholecalciferol was significantly lower in patients with psoriasis than in healthy individuals, and the lowest measure was obtained from patients with severe psoriasis. These results correlate with similar observations of other authors. Both the studies of Ricceri et al. and Orgaz-Molina et al. revealed a high prevalence of insufficiency and deficiency in serum 25-hydroxycholecalciferol, which was greater in patients with psoriasis than in control subjects [15, 16]. Additionally, comparatively to our observations, significantly lower values of 25-hydroxycholecalciferol were reported by Ricceri et al., and these concentrations negatively correlated with PASI [15]. An interesting observation was made in the study of Orgaz-Molina et al. who reported a higher risk of 25-hydroxycholecalciferol insufficiency in psoriatic patients with a body mass index of 27 or more. A possible explanation for such a relationship was the lower physical activity level and therefore reduced sun exposure of heavier psoriatic patients. Another possible mechanism is the increased sequestration of vitamin $D_{3}$ in fat, which could result in its reduced bioavailability in serum [16].

The main source of vitamin $D_{3}$ is its cutaneous synthesis, which is activated by UV radiation; however, the safe dose of exposure to UV radiation, which does not cause the development of skin cancer, has remained controversial. The current trends in lifestyle involve only minimal sun exposure through most of the year but with excessive sunbathing during the holiday period, which undoubtedly implies a risk for the development of cutaneous neoplasia, including melanoma [1, 2, 9, 17, 18].

The impact of light exposure on the synthesis of 25-hydroxycholecalciferol has been evaluated in several clinical studies. Ala-Houhala et al. analyzed the influence of narrow-band ultraviolet B (UVBnb) phototherapy on the serum level of 25-hydroxycholecalciferol and the severity of skin lesions in patients with psoriasis. In that study, the response to UVBnb was assessed in $12 \mathrm{pa}$ tients who were concomitantly supplemented with oral cholecalciferol, $20 \mu \mathrm{g}$ daily. At baseline, the serum level of 25-hydroxycholecalciferol was $74.14 \mathrm{nmol} / \mathrm{l}$ (much higher than in our study), and it increased by $49.4 \mathrm{nmol} / \mathrm{l}$ above baseline at the $18^{\text {th }}$ exposure of UVBnb [11]. In another study, Feldmeyer et al. compared the differences in serum concentrations of 25-hydroxycholecalciferol which occurred after phototherapy with different light spectra. The group of 116 dermatologic patients included several disease entities (atopic dermatitis, psoriasis, morphea, and others), and all individuals underwent UVA1 $(n=38)$, UVBnb $(n=48)$ or combined UVA/UVBnb $(n=30)$ phototherapy with the frequency of 2 to 3 times a week for 53 to 90 days. It was observed that phototherapy with UVBnb and UVA/ UVBnb increased the 25-hydroxycholecalciferol serum level significantly, whereas UVA1 therapy alone induced a reduction in serum 25-hydroxycholecalciferol concentrations [19].

A considerable source of vitamin $D_{3}$ that enables limitation of sun exposure seems to be its additional food intake, in the form of certain foods or dietary supplements. Unfortunately, most meals contain only a little vitamin $D_{3}$, and those rich in vitamin $D_{3}$ are eaten irregularly, which may be an important explanation of why both study groups do not consume adequate quantities of vitamin $D_{3}[1,2,9,20,21]$. In a pilot study, Finamor et al. observed that prolonged high-dose vitamin $D_{3}$ administration could be very beneficial for patients with psoriasis. It was found that treatment with vitamin $D_{3}(35,000$ IU daily) resulted in a significant increase in serum level of 25-hydroxycholecalciferol, which correlated with a significant improvement in the PASI score of all patients. Essentially, all patients presented low vitamin D status (serum 25 -hydroxycholecalciferol $\leq 30 \mathrm{ng} / \mathrm{ml}$ ) at baseline, which is in agreement with our results [22].

The benefit/risk ratio should be taken into account, since the sun exposure is undoubtedly crucial in supporting an adequate level of vitamin $\mathrm{D}_{3}$. Overall, moderate sun exposure throughout the year and a balanced diet should be the recommended practice to support a sufficient amount of vitamin $D_{3}$ metabolites with maintenance of the serum 25-hydroxycholecalciferol within reference limits. In addition, new studies that determine both the intake and blood levels of 25-hydroxycholecalciferol in psoriasis patients are required $[1,2,23]$.

\section{Conclusions}

The considerably low serum content of vitamin $D_{3}$ in both psoriasis patients and the control group indicate the need for more research to evaluate the vitamin $D_{3}$ status of our population. 
It is necessary to bear in mind that not only meals rich in vitamin D, but also sun exposure, are essential to maintain an adequate level of this vitamin.

Although the food intake of vitamin $D_{3}$ is very important for both the general population and psoriasis patients, these groups manifest altered metabolism of vitamin $D_{3}$ and, in consequence, different vitamin $D_{3}$ requirements.

\section{Acknowledgments}

This study was funded by statutory grant KNW-1119/N/4/0.

\section{Conflict of interest}

The authors declare no conflict of interest.

\section{References}

1. Suárez-Varela MM, Reguera-Leal P, Grant WB, et al. Vitamin $\mathrm{D}$ and psoriasis pathology in the Mediterranean region, Valencia (Spain). Int J Environ Res Public Health 2014; 11: 12108-17.

2. Leyssens $C$, Verlinden L, Verstuyf $A$. The future of vitamin $D$ analogs. Front Physiol 2014; 5: 122.

3. Millsop JW, Bhatia BK, Debbaneh M, et al. Diet and psoriasis, part III: role of nutritional supplements. J Am Acad Dermatol 2014; 71: 561-9.

4. Finamor DC, Sinigaglia-Coimbra R, Neves LC, et al. A pilot study assessing the effect of prolonged administration of high daily doses of vitamin D on the clinical course of vitiligo and psoriasis. Dermatoendocrinol 2013; 5: 222-34.

5. Slominski A, Kim TK, Zmijewski MA, et al. Novel vitamin D photoproducts and their precursors in the skin. Dermatoendocrinol 2013; 5: 7-19.

6. Datta Mitra A, Raychaudhuri SP, Abria CJ, et al. 1alpha,25dihydroxyvitamin-D3-3-bromoacetate regulates AKT/mTOR signaling cascades: a therapeutic agent for psoriasis. J Invest Dermatol 2013; 133: 1556-64.

7. Chiang KC, Yeh CN, Hsu JT, et al. Evaluation of the potential therapeutic role of a new generation of vitamin D analog, MART-10, in human pancreatic cancer cells in vitro and in vivo. Cell Cycle 2013; 12: 1316-25.

8. Morimoto S, Kumahara Y. A patient with psoriasis cured by 1 alpha-hydroxyvitamin D3. Med J Osaka Univ 1985; 35: 51-4.

9. Macejova D, Ondkova S, Jakubikova L, et al. MNU-induced mammary gland carcinogenesis: chemopreventive and therapeutic effects of vitamin D and Seocalcitol on selected regulatory vitamin D receptor pathways. Toxicol Lett 2011; 207: 60-72.

10. Wu-wong JR, Tian J, Golzman D. Vitamin D analogs as therapeutic agents: a clinical study update. Curr Opin Investing Drugs 2004; 5: 320-6.

11. Ala-Houhala MJ, Karppinen T, Vähävihu K, et al. Narrow-band ultraviolet B treatment boosts serum 25 -hydroxyvitamin D in patients with psoriasis on oral vitamin D supplementation. Acta Derm Venereol 2014; 94: 146-51.

12. Okita H, Ohtsuka T, Yamakage A, Yamazaki S. Polymorphism of the vitamin D(3) receptor in patients with psoriasis. Arch Dermatol Res 2002; 294: 156-62.
13. Ahn CS, Awadalla F, Huang KE, et al. Patterns of vitamin D analog use for the treatment of psoriasis. I Drugs Dermatol 2013; 12: 906-10.

14. Griffin MD, Xing N, Kumar R. Gene expression profiles in dendritic cells conditioned by 1alpha,25-dihydroxyvitamin D3 analog. J Steroid Biochem Mol Biol 2004; 89-90: 443-8.

15. Ricceri F, Pescitelli L, Tripo L, Prignano F. Deficiency of serum concentration of 25-hydroxyvitamin D correlates with severity of disease in chronic plaque psoriasis. J Am Acad Dermatol 2013; 68: 511-2.

16. Orgaz-Molina J, Buendía-Eisman A, Arrabal-Polo MA, et al. Deficiency of serum concentration of 25-hydroxyvitamin D in psoriatic patients: a case-control study. J Am Acad Dermatol 2012; 67: 931-8.

17. Kudish Al, Harari M, Evseev EG. The measurement and analysis of normal incidence solar UVB radiation and its application to the photoclimatherapy protocol for psoriasis at the Dead Sea, Israel. Photochem Photobiol 2011; 87: 217-22.

18. Menter A, Gold LS, Bukhalo M, et al. Calcipotriene plus betamethasone dipropionate topical suspension for the treatment of mild to moderate psoriasis vulgaris on the body: a randomized, double-blind, vehicle-controlled trial. J Drugs Dermatol 2013; 12: 92-8.

19. Feldmeyer L, Shojaati G, Spanaus KS, et al. Phototherapy with UVB narrowband, UVA/UVBnb, and UVA1 differentially impacts serum 25-hydroxyvitamin-D3. J Am Acad Dermatol 2013; 69: 530-6.

20. Katz U, Shoenfeld Y, Zakin V, et al. Scientific evidence of the therapeutic effects of dead sea treatments: a systematic review. Semin Arthritis Rheum 2012; 42: 186-200.

21. Li YC. Vitamin D regulation of the renin-angiotensin system. J Cell Biochem 2003; 88: 327-31.

22. Khandpur S, Bhari N. Newer targeted therapies in psoriasis. Indian J Dermatol Venereol Leprol 2013; 79: 47-52.

23. Akasaka E, Mabuchi T, Manabe Y, et al. Long-term efficacy of psoriasis vulgaris treatments: analysis of treatment with topical corticosteroid and/or vitamin D3 analog, oral cyclosporin, etretinate and phototherapy over a 35 -year period, 1975-2010. J Dermatol 2013; 40: 238-43. 